\title{
DEVELOPMENT, CHARACTERIZATION \& STABILIZATION OF POORLY WATER SOLUBLE DRUGS UTILIZING SOLID DISPERSION TECHNIQUES BY USING B - CYCLODEXTRIN
}

\author{
Rakesh Singh*1, Dr D. A. Jain², \\ Institue of Pharmaceutical Science and Research Centre; Bhagwant University, Ajmer \\ *Corresponding author's Email: rakesh01py40@yahoo.co.in
}

\begin{abstract}
Telmisartan (TLM) is an angiotensin II receptor antagonist used in the treatment of hypertension. According to BCS (biopharmaceutical classification system) Telmisartan belongs to class II drug, and it is practically insoluble in water and it shows low dissolution profile and poor absorption. The present study is to improve the solubility of Telmisartan by forming complexation with $\beta$ - CD by using four convenient methods viz physical mixing method, kneading method, and solvent evaporation fusion method at different molar ratios of 1:1, dissolution studies were carried out in $\mathrm{pH} 7.4$ phosphate buffer. The cyclodextrin complexes formulated by employing 1:1 (drug: complexing agent) with kneading technique showed higher drug release.

Keywords: Telmisartan, inclusion complex, $\beta$ - cyclodextrin, physical, kneading, solvent evaporation \& fusion method.
\end{abstract}

\section{INTRODUCTION}

By many estimates up to 40 percent of new chemical entities (NCEs) discovered by the pharmaceutical industry today are poorly soluble or lipophilic compounds. The solubility issues complicating the delivery of these new drugs also affect the delivery of many existing drugs. The ability to deliver poorly soluble drugs will grow in significance in the coming years as NCEs are relied upon for a larger share of the revenue within the pharmaceutical market by innovator companies. Similarly, generic drug manufacturers will need to employ economically efficient methods of delivery as more low solubility drugs go off patent, in order to maintain a competitive edge and sufficiently compete as profit margins shrink in this pricesensitive industry. Relative to highly soluble compounds, low drug solubility often manifests itself in a host of in vivo consequences, including decreased bioavailability, These in vivo and in vitro characteristics and the difficulties in achieving predictable and reproducible in vivo/in vitro correlations are often sufficiently formidable to halt development on many newly synthesized compounds due to solubility issues. Relative to highly soluble compounds, low drug solubility often manifests itself in a host of in vivo consequences, including decreased bioavailability. Poorly soluble compounds also present many in vitro formulation obstacles, such as severely limited choices of delivery technologies and increasingly complex dissolution testing with limited or poor correlation to the in vivo absorption. These in vivo and in vitro characteristics and the difficulties in achieving predictable and reproducible in vivo/in vitro correlations are often sufficiently formidable to halt development on many newly synthesized compounds due to solubility issues. The Biopharmaceutical Classification System (BCS) groups poorly soluble compounds as Class II and IV drugs, compounds which feature poor solubility and high permeability, and poor solubility and poor permeability, respectively. Drug substances are considered highly soluble when the largest dose of a compound is soluble in $<250 \mathrm{~mL}$ water over a range of $\mathrm{pH}$ from 1.0 to 7.5; highly permeable compounds are classified as those compounds that demonstrate $>90$ percent absorption of the administered dose (1). In contrast, compounds with solubility below $0.1 \mathrm{mg} / \mathrm{mL}$, and often-even compounds with solubility below $10 \mathrm{mg} / \mathrm{mL}$ present difficulties related to solubilisation during formulation. ${ }^{1}$

Cyclodextrins (CDs), with their ability to form molecular inclusion complexes with drug substances affect many of the physic chemical properties of the drugs without affecting their intrinsic lipophilicity or pharmacological properties As a consequence of the inclusion process,many physicochemical properties, such as solubility, dissolution rate, stability, palatability, and bioavailability, can be favourably affected CDs are thus offering new hope to formulation scientists in their efforts to develop an effective drug delivery system.

The number of applications of CDs in pharmaceutical formulations has been increasing in recent years because of their approval by various regulatory agencies However; the use of CDs in solid oral dosage forms is limited to lowdose drugs with large stability constants because of the mass limitations of oral dosage units.

Cyclodextrins (CDs) improve solubility significantly they are still limited in their drug inclusion capacity and retain disadvantageous processing characteristics for oral dosage forms; the volume of CD complexes is often much greater than the volume of drug alone, which may severely limit the types of delivery technologies that may be employed. ${ }^{2}$

CD complexes have also been employed in conjunction with hydrophilic polymers, such as hydroxylpropylmethyl cellulose, to improve the solubilising effect of the CDs. 
The improvement in solubilisation ability within these water-soluble polymer/drug included CD aggregates requires less cyclodextrin to solubilise the same amount of drug, reducing the volume constraints present for non aggregated $\mathrm{CDs}$ and increasing the range of delivery technologies available.

\section{MATERIALS AND METHODS:}

Pure sample of Telmisartan was obtained from Medley Pharma limited, Daman. $\beta-C D$ is purchased from SD Fine Chemicals Mumbai.

\section{Methods of preparation:}

1. Dry / Physical mixing: Some guests can be complexed by simply adding the guest to the CD and mixing/triturating them together. This works best with oils or liquid guests.

2. Kneading Method: $\beta$ - CD was mixed in glass mortar along with water to obtain a homogeneous paste. The drug was then slowly added to the paste and the mixture was triturated for $1 \mathrm{hr}$. during the process the water content was empirically adjusted to maintain the consistency of the paste. The paste formed was dried under vacuum for 24 hours. Dried powder was passed through specific sieve no. and stored in a dessicator until further evaluation. ${ }^{3}$

3. Solvent Method: Researchers often use hybrid fusionsolvent method if thermal instability and immiscibility between the compound(s) and the carrier are present. In the process, the researchers first dissolve the compound in a small quantity of organic solvent and added to the molten carrier. Researchers then evaporate the solvent to generate the mass. They mill this mass to produce powder at desired particle size ranges.

4. Fusion-melt Method: The fusion-melt involves melting the compound(s) and the carrier components together at temperatures at or above the melting point of all components. In the fusion process, researchers blend the compound and carrier in a suitable mixer. They heat, melt the blend and then cool the molten mixture rapidly to provide a congealed mass. They mill this mass to produce powders at desired particle size ranges

\section{Experimental Methods:}

Spectral and absorbance measurements by using UV Visible spectrophotometer by using,1-cm quartz cells. A simple UV spectrophotometric method was developed for the determination of Telmisartan in pure and its pharmaceutical formulations.

Telmisartan exhibited maximum absorbance at $296 \mathrm{~nm}$ in Phosphate buffer ( $\mathrm{pH}$ 7.4) Containing Sodium lauryl sulphate $(0.2 \%)$ and obeyed linearity in the concentration range of $1-10 \mu \mathrm{g} / \mathrm{ml}$.

\section{Preparation of Stock Solution:}

Standard stock solution of Telmisartan was prepared by dissolving $10 \mathrm{mg}$ of drug in $100 \mathrm{ml}$ of Phosphate buffer (pH 7.4) Containing Sodium lauryl sulphate $(0.2 \%)$ in 100 $\mathrm{ml}$ of volumetric flask to get a concentration of $10 \mu \mathrm{g} / \mathrm{ml}$.
Preparation of Working Standard Solutions and construction of standard graph:

To construct Beer's law plot for Telmisartan, the stock solution was further used to prepare working standard solutions of concentrations ranging from 1 to $10 \mu \mathrm{g} / \mathrm{ml}$ different aliquots of working standard solutions of Telmisartan was transferred separately into a series of 10 $\mathrm{ml}$ volumetric flasks and diluted to $10 \mathrm{ml}$ using phosphate buffer .The absorbance were measured at $\lambda \max 296 \mathrm{~nm}$ against buffer as blank. The standard graph for Telmisartan was plotted by taking concentration of drug on $\mathrm{x}$-axis and absorbance on $y$-axis and is shown in fig 1. The drug has obeyed Beer's law in the concentration range of 1-10 $\mu \mathrm{g} / \mathrm{ml}^{4}$

\section{Fourier Transforms Infrared (FTIR) spectroscopy.}

FTIR Spectroscopy was performed on Lab India by scanning the sample in zink seleniume (Znse). Before taking the spectrum of the sample, a blank spectrum of air background was taken. Number of scans, 24; resolution, 4 $\mathrm{cm}-1$; range, $500-4000 \mathrm{~cm}^{-1}$ The sample of Pure Drug, and $\boldsymbol{\beta}$ - Cyclodextrin were scanned. The complexes of $\boldsymbol{\beta}$ CD with TEL prepared by different methods were scanned by FTIR ranges from 500-4000, There is no interaction between drug \& beta CD. (Figure 5).

\section{Powder x-ray diffractromety:}

Powder X-ray diffraction patterns were recorded on X-Ray diffraction instrument (Philips Analytical X'Pert PRO) with $\mathrm{Cu}$ radiation, at a voltage of $45 \mathrm{kV}$ and current of $40 \mathrm{~mA}$. The scanning speed was Gonio between 5 and 40theta. diffraction angle $(2 \theta)$ range.

\section{RESULT \& DISCUSSION:}

The linear relationship between the concentration of Telmisartan and the corresponding absorbance values was shown by- $\mathrm{Y}=0.055 \mathrm{X}+0.011$ Where, $\mathrm{Y}=$ absorbance, and $X=$ concentration of Telmisartan $(\mu \mathrm{g} / \mathrm{ml})$ A positive correlation between the concentration of Telmisartan and the corresponding absorbance values was observed (correlation coefficient, $r^{2}=0.993$ ). The amount of Telmisartan in either the $\beta$-CD complex or the dissolution fluids was calculated using the linear relationship as given above or directly from the standard graph as shown in fig 1 .

\section{Preparation of solid complexes}

Complexes of $\boldsymbol{\beta}$-CD with TEL were prepared in the molar ratio of $1: 1$ (on the basis of phase solubility study) by different methods like Physical mixing, Kneading, Solvent evaporation, and Fusion method.).

\section{Physical Mixture:}

Physical mixture was prepared by triturating TEL and $\beta$ $\mathrm{CD}$ together for $30 \mathrm{~min}$ in a clean and dry glass mortar until a homogeneous mixture was obtained. And then was forced through sieve no 100 . 


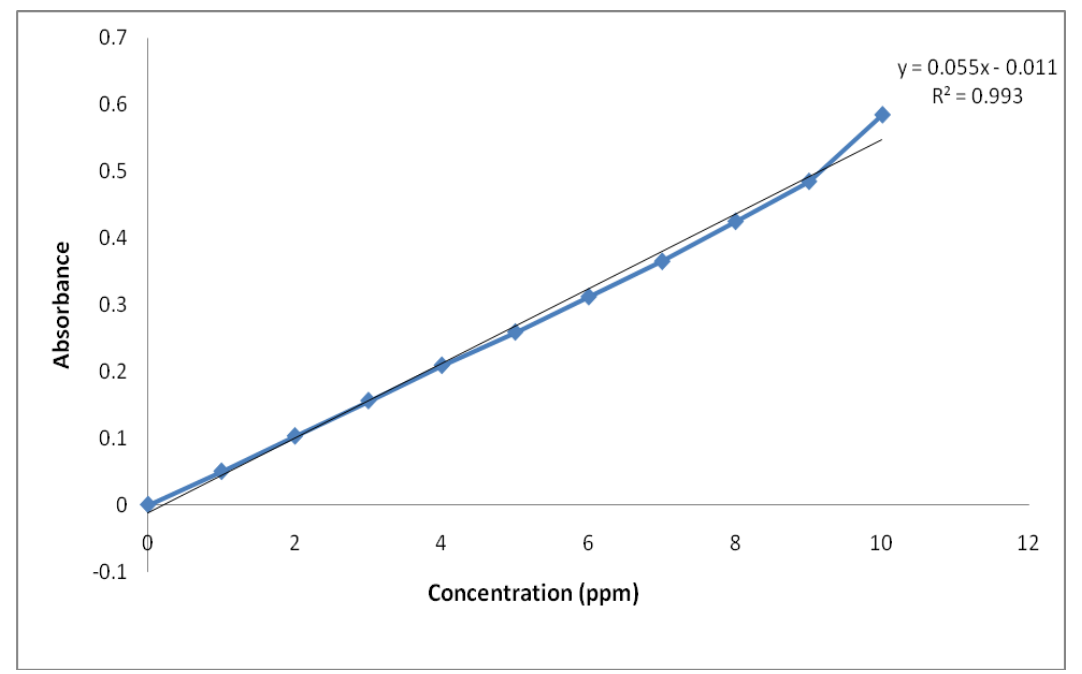

Figure 1: Standard curve for the estimation of Telmisartan in phosphate buffer pH 7.4

\section{Kneading Method:}

$\boldsymbol{\beta}$-CD mixed in glass mortar along with water to obtain a homogeneous paste. The drug (either in powder form or as solution with minimum quantity of methanol) was then slowly added to the paste and the mixture was triturated for $1 \mathrm{hr}$. during the process the water content was empirically adjusted to maintain the consistency of the paste. Methanol was added to assist dissolution of TEL during the process. The paste was dried at room temp., pulverized and forced through sieve no $100 .^{5}$

\section{Fusion Method:}

TEL and $\boldsymbol{\beta}$-CD were thoroughly mixed and placed in a sealed container with a small amount of water. The contents are heated to about $100^{\circ} \mathrm{C}$ and then removed and dried. The mass was then pulverized and forced through sieve no 100.

\section{Solvent evaporation Method: ${ }^{7,8}$}

A solution of Telmisartan in methanol was gradually added to equi-molar concentration of $\boldsymbol{\beta}$-CD in water and agitated at $50^{\circ} \mathrm{C}$ for $30 \mathrm{~min}$ and toward the end of addition turbidity developed in the mixture. At the end of this period the solution was filtered, and the moist solid was kept in oven $50^{\circ} \mathrm{C}$ for removal of last trace of solvent. The mass was then pulverized and passed through sieve no 100.

Table 1: Preparation of Telmisartan \& $\beta$-CD solid dispersions by different technique

\begin{tabular}{|l|l|l|l|}
\hline Type of formulation & TEL: $\beta$-CD (molar ratio) & Solid dispersion Method & Media \\
\hline TPM & $1: 1$ & Physical Mixing & $\ldots \ldots \ldots$ \\
\hline TKW & $1: 1$ & Kneading & Water \\
\hline TKM & $1: 1$ & Kneading & Methanol + Water \\
\hline TSE & $1: 1$ & Solvent Evaporation & Methanol + Water \\
\hline TFW & $1: 1$ & Fusion & Water \\
\hline
\end{tabular}

Table 2: Drug content of Tel $\beta-C D$ complexes (\% Drug content)

\begin{tabular}{|l|l|l|l|}
\hline Formulation & Theoretical drug content in 100mg & Practical drug content in 100mg (mean n=3) & Drug content \\
\hline TPM & 27.64 & 27.02 & 97.75 \\
\hline TKW & 27.64 & 26.93 & 97.43 \\
\hline TKM & 27.64 & 27.12 & 98.12 \\
\hline TSE & 27.64 & 27.08 & 97.98 \\
\hline TFU & 27.64 & 27.11 & 98.08 \\
\hline
\end{tabular}

Saturation solubility of different formulations of Telmisartan.

The saturation solubility of pure TEL and its complexes with $\beta$-CD is shown in table 3 . The saturation solubility of pure TEL is $11.9 \mu \mathrm{g} / \mathrm{ml}$ while the saturation solubility of all other complex prepared by various methods exhibited dramatic increase in the saturation solubility. TPM and $\beta$ CD (complex prepared by physical mixing) showed a lower value for saturation solubility than that of other complexes, the low saturation solubility can be attributed to poor complexation efficiency during physical mixing. 
Fourier Transforms Infrared (FTIR) spectroscopy.

FTIR spectra of TEL and $\beta-$ CD its Complex are presented In (Figure 5). Pure Telmisartan spectra showed sharp characteristic peaks at 3746, 2958, 1693, 1456 and $1266 \mathrm{~cm}-1$ All the above characteristic peaks appears in the spectra of all Complex at same wavenumber indicating no modification or interaction between the drug and $\beta-$ CD. .

\section{Powder x-ray diffractromety:}

Powder x-ray diffraction (XRD) of Pure Drug \& $\beta-C D$ with complex show in (Figure 8.) The $X$ - ray diffractogram of Telmisartan has sharp peak at different angle (2ө) $6.72^{\theta}, 14.17^{\theta}, 18.97^{\theta}, 22.18^{\theta}, 25.85^{\theta}$ show a tripical crystalline pattern. However, all major characteristic crystalline peaks appear in the diffractogram of both physical mixtures and solid dispersion system. Moreover, the relative intensity and $2 \theta$ angle of these peaks remains practically unchanged. Thus it can be clearly suggestive from X-ray data that there is no amorphization of TEL. and it is still in its original crystalline form.

\section{Characterization of complexation:}

Table: 3. Saturation solubility of different formulations of Telmisartan.

\begin{tabular}{|c|c|}
\hline Formulation & Saturation solubility of Telmisartan \\
\hline Pure TEL & $11.95 \pm 0.84$ \\
\hline TPM & $78.90 \pm 2.11$ \\
\hline TKW & $135.78 \pm 2.31$ \\
\hline TKM & $141.47 \pm 2.67$ \\
\hline TSE & $104.99 \pm 2.67$ \\
\hline TFU & $119.82 \pm 2.48$ \\
\hline
\end{tabular}

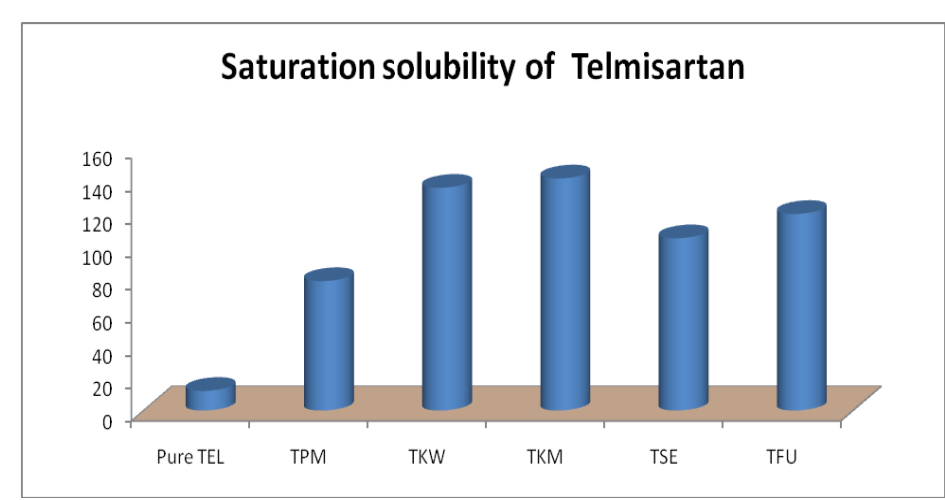

Figure 2: Saturation solubility of Telmisartan

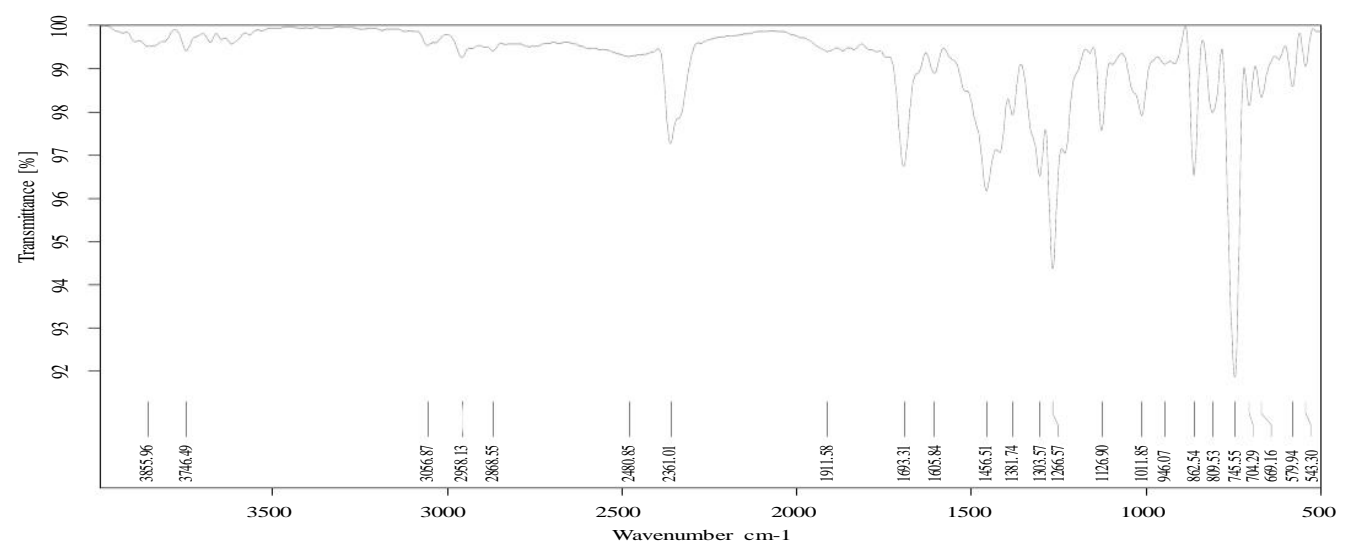

Figure 3: FTIR spectrum of pure Drug Telmisartan

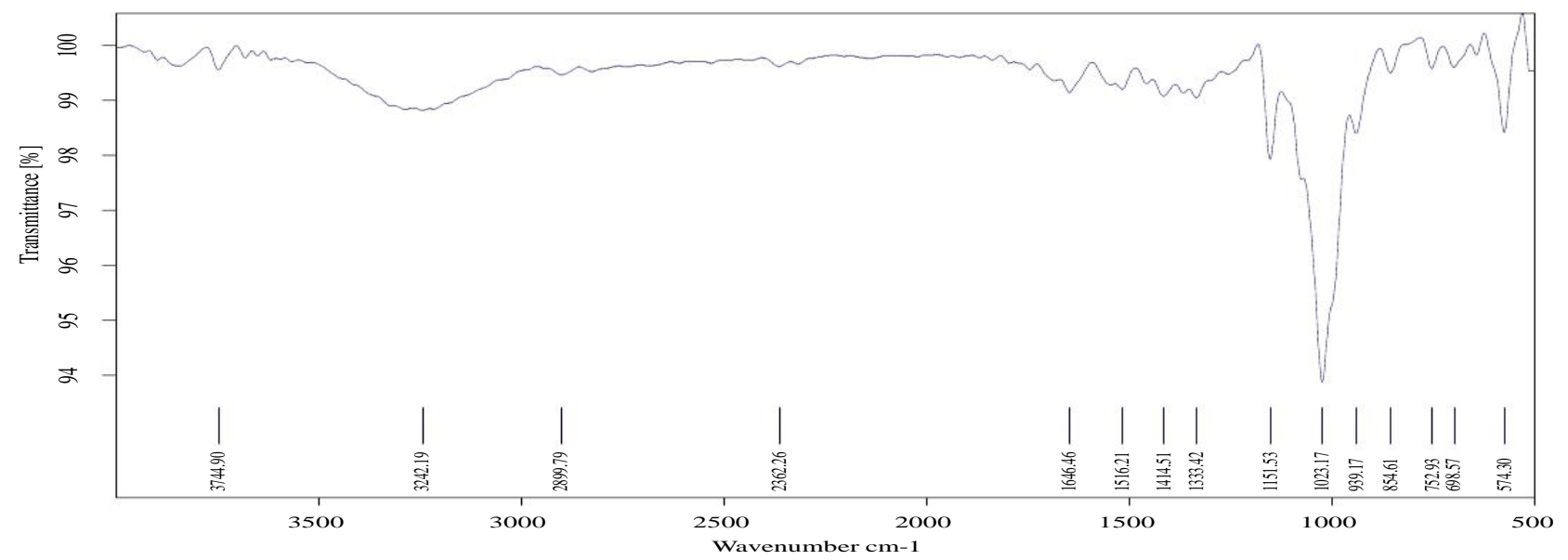

Figure 4: F'IIR spectrum of pure $\beta$ - cyclodextrin. 


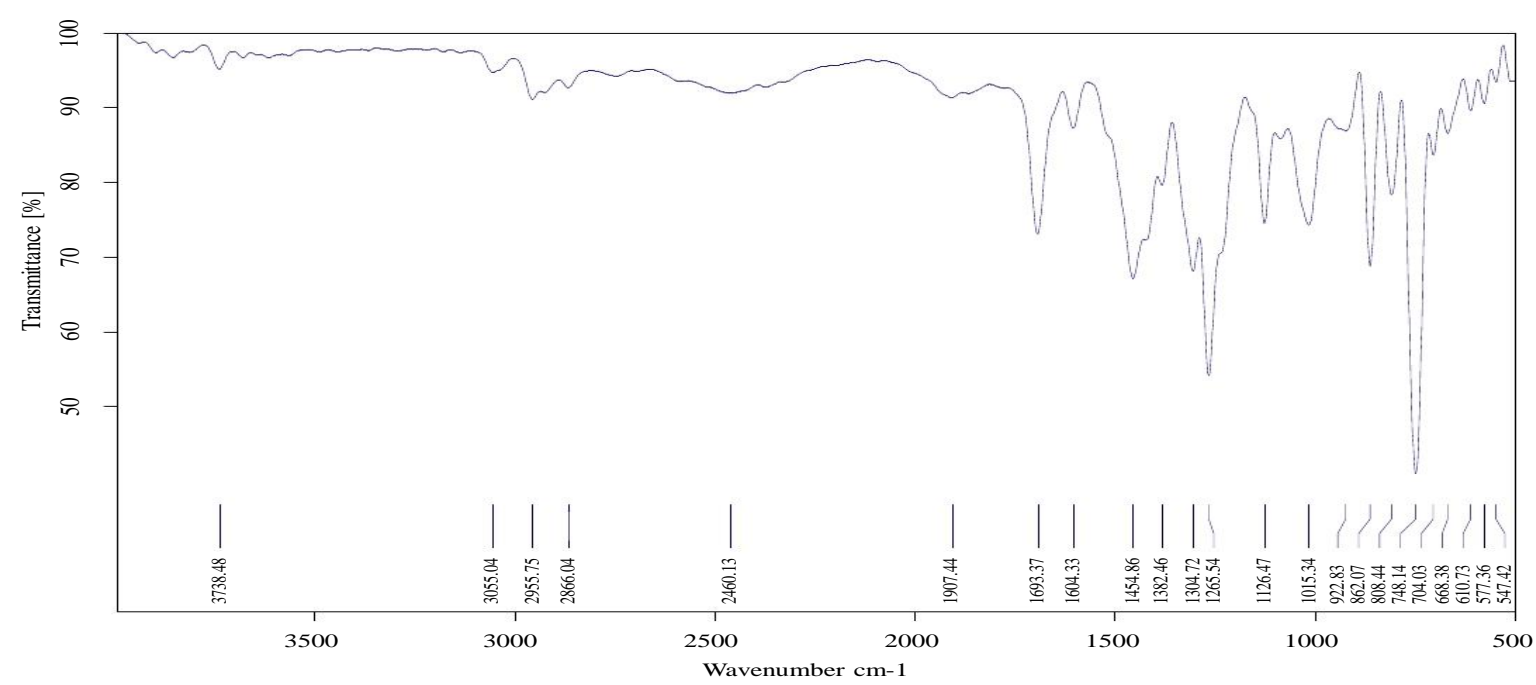

Figure 5: FTIR Spectrum of mixture of (TEL\& $\beta$--CD)

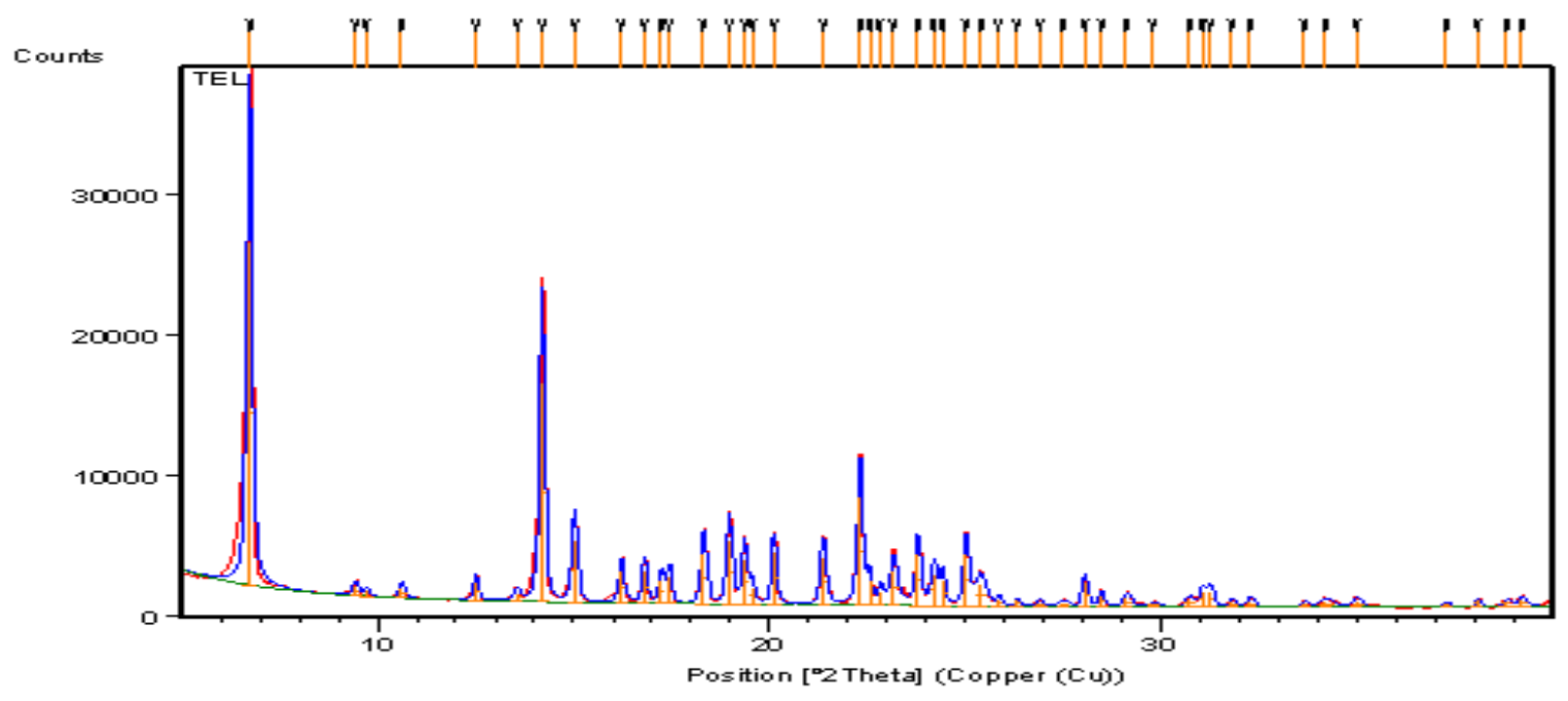

Figure 6: x-ray diffractromety of Pure Telmisartan..

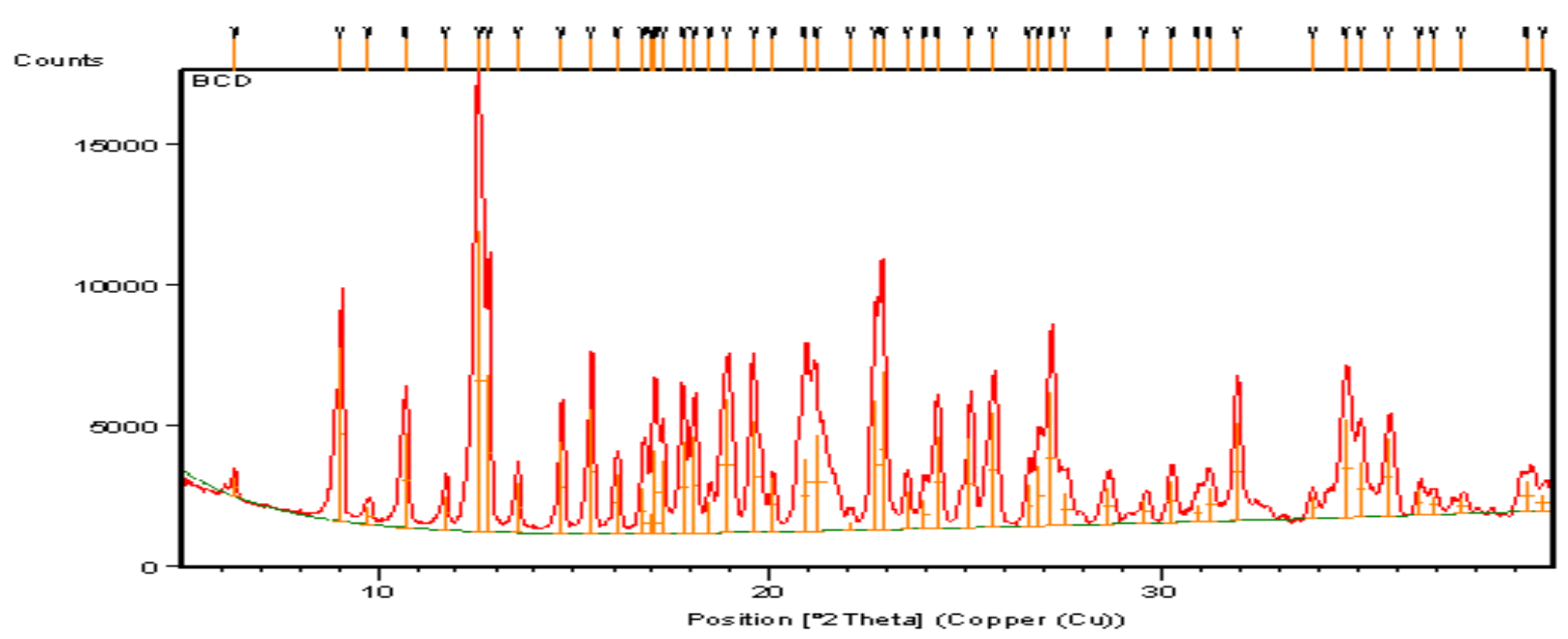

Figure 7: x-ray diffractromety of $\beta$ - CD 


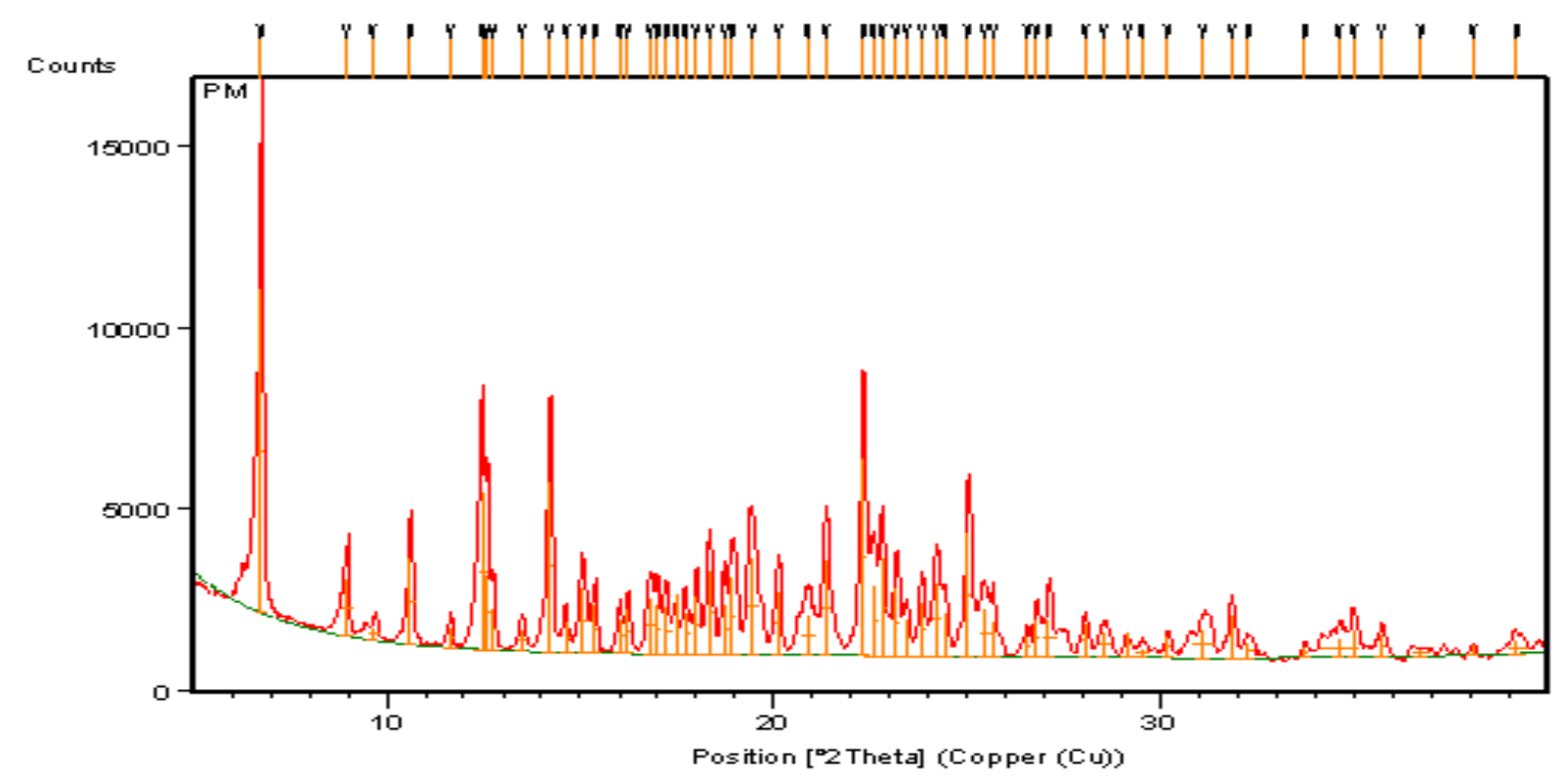

Figure 8: x-ray diffractromety of Mixture Tel + $\beta$ - CD.

\section{CONCLUSION}

Solid dispersions of Telmisartan were prepared by Different technique of solid dispersion method using carrier's $\beta$ - CD. In the present work total five formulations were prepared by using Telmisartan with $\beta$ - CD by using five convenient methods viz physical mixing method, kneading method, and solvent evaporation fusion method at different molar ratios of $1: 1$, dissolution studies were

\section{REFERENCES}

1. Michael Hite, Stephen Turner, Cathy Federici, SCOLR Inc; Oral delivery of poorly soluble drug. Pharmaceutical Manufacturing and Packing Sourcer Summer '03 issue, Samedan Ltd.

2. Fromming $\mathrm{KH}$, Szejtli J. CDs in Pharmacy. Dordrecht, The Netherlands: Kluwer Academic; 1994.

3. Duchene D, Wouessidjewe D. Pharmaceutical and medicinal applications of cyclodextrins. In: Dumitriu S, ed. Polysaccharides in Medical Applications. New York, NY: Marcel Dekker; 1996: 575-602.

4. Uekama K, Hirayama F, Irie T. Cyclodextrin drug carrier systems. Chem Rev. 1998; 98:2045-2076.

5.Kora Pattabhi Ramaiah Chowdary and Sekuboyina Vijaya Srinivas; Influence of Hydrophilic Polymers on Celecoxib Complexation with Hydroxypropyl $\beta$-Cyclodextrin. AAPS PharmSciTech 2006; 7 (3) Article 79.

6 .M.Narender Reddy, Tasneem Rehana, S. Ramakrishna, et al;Cyclodextrin Complexes of Celecoxib:Molecular-Modelling carried out in $\mathrm{pH} 7.4$ phosphate buffer. The cyclodextrin complexes formulated by employing 1:1 (drug: complexing agent) with kneading technique showed higher drug release. 9,1

\section{ACKNOWLEDGMENTS}

The authors acknowledge for Bhagwant University \& also thank to Medley pharma Daman they provide gift sample for project work.

Characterization, and Dissolution Studies. AAPS PharmSci 2004; 6 (1) Article 7.

7. Thorsteinn Loftsson, Dagny Hreinsdottir, Mar Masson; Evaluation of cyclodextrin solubilization of drugs. International Journal of Pharmaceutics 302 (2005) 18-28.

8. Aftab Modi and Pralhad Tayade Enhancement of Dissolution Profile by Solid Dispersion (Kneading) Technique. AAPS PharmSci 2004; 6 (1) Article 7(3)2006,

9. Gerrit S. Zijlstra Characterization of a Cyclosporine Solid Dispersion For Inhalation.

AAPS PharmSci. 2007 June; 9(2) 2007

10.Melleswara Rao V.S.N, Shyam T, \& Srinivasa Raos Y, Formulation and characterization of meloxicam solid dispersions, Int. J. Pharm., 2007, 34-39.

11.Marin MT, Margarit MV, Salcedo. Characterization and solubility study of solid dispersions of flunarizine and polyvinylpyrrolidone. Int.J.Pharm. 2002; 57:72.

12.http://en.wikipedia.org/wiki/Telmisartan. 